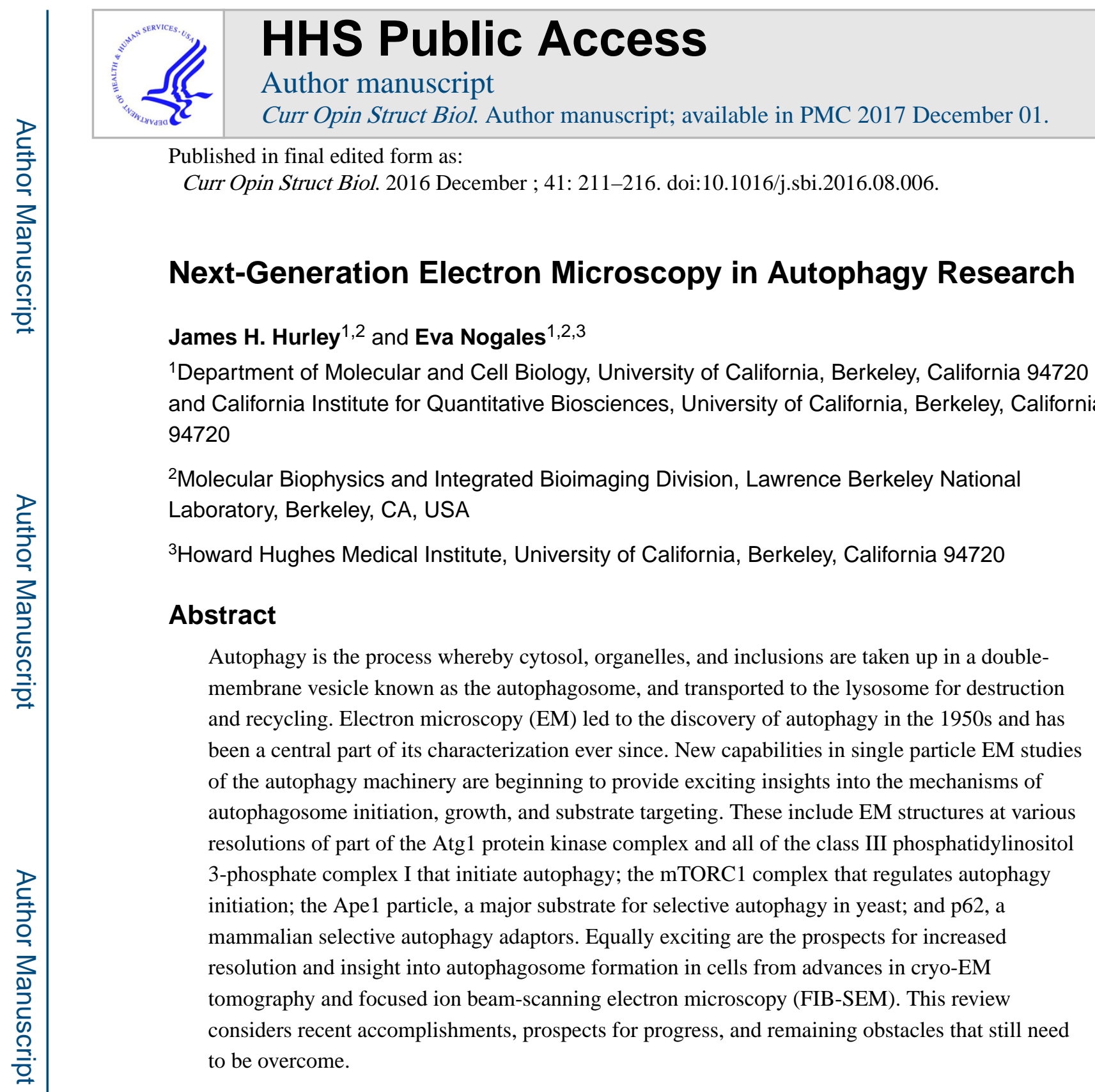

\title{
Introduction
}

Autophagy is a conserved process of eukaryotes whereby cellular contents are engulfed in a double membrane vesicle and degraded in the lysosome (1). It in most ancient form, autophagy is a mechanism for cell survival in starvation. Starvation-induced autophagy typically involves the bulk and seemingly indiscriminate uptake of cytosolic contents. In multicellular organisms, the main function of autophagy seems to be to "clear out the junk" from the cytosol. Protein inclusions, depolarized mitochondria, intracellular pathogens and other unneeded or even harmful materials are removed by autophagy. Typically this form of

\footnotetext{
Correspondence: enogales@lbl.gov or jimhurley@berkeley.edu.

Publisher's Disclaimer: This is a PDF file of an unedited manuscript that has been accepted for publication. As a service to our customers we are providing this early version of the manuscript. The manuscript will undergo copyediting, typesetting, and review of the resulting proof before it is published in its final citable form. Please note that during the production process errors may be discovered which could affect the content, and all legal disclaimers that apply to the journal pertain.
} 
autophagy is targeted and is referred to as "selective" autophagy $(2,3)$. When this clearance mechanism fails, disease can ensue. Neurodegeneration is one of the most striking consequences of autophagic failure, and Parkinson's disease and others have been linked to autophagic defecits (4).

Autophagy was discovered in the late 1950s through 1960s, the early years of transmission electron microscopy (TEM) of fixed cells (5). Early electron microscopy (EM) studies revealed striking images of, for example, mitochondria sequestered within the larger vesicular structures now known as autophagosomes. EM was the main tool for studying autophagy until genetics came to the fore in the 1990s and fluorescence microscopy in the 2000s. Thanks to yeast genetics, the components of the molecular machinery of autophagy are now largely known (1). Yet the detailed workings of this machinery in autophagosome biogenesis are still among the more mysterious events in cell biology (6). The roles of autophagy in a host of medically important processes, from cancer to infection to neurodegeneration, have led to a redoubled interest in the molecular mechanisms of autophagy.

This intense interest in the molecular and cellular underpinnings of autophagy has coincided with a revolution in the capabilities of EM methodologies, both those aiming to gain structural information at high resolution of purified components (i.e. single particle 3D reconstruction), as well as those used to characterize cellular ultrastructure (e.g. electron tomography or FIB-SEM (focused ion beam-scanning electron microscopy)). Single particle cryo-EM is a powerful structural biology technique that overcomes the need for crystallization and requires only minute amounts of sample, properties that make it of very broad applicability. Recent technological breakthroughs in cryo-EM, specially concerning detector technology and computational image processing, have resulted in a burst of threedimensional structures of essential macromolecules of very high biological relevance that had for long resisted structural characterization, many of them at near-atomic resolution (7). Cryo-EM tomography of cells or organelles is also gaining in applicability and resolution, thanks to technological advances such as FIB-milling to generate windows into the cellular content or the application of subtomogram averaging to obtain unprecedented resolution. Methods like FIB-SEM are pushing for the largest real state, allowing modest resolution but of whole cells or tissue samples. These new developments have only just begun to contribute to autophagy research. The purpose of this review is two-fold: both to summarize the early contributions of this new wave of EM studies to our understanding of autophagy, and to highlight the unrealized potential of these methods to bring autophagy research to a higher level of insight.

\section{The core machinery of autophagy}

A handful of protein complexes drive autophagosome biogenesis in both bulk and selective autophagy. Two of these have been studied by single particle EM. The ULK1/Atg1 complex $(8,9)$ consists of the protein kinase ULK1 (Atg1 in yeast), and the non-catalytic subunits Atg13, FIP200 (Atg11 or Atg17 in yeast), Atg101 (except in budding yeasts), and Atg29 and Atg31 (only in budding yeast). The class III phosphatidylinositol 3-kinase complex I 
(PI3KC3-C1) consists of the lipid kinase subunit Vps34 and the scaffolding and regulatory subunits Vps15, Beclin-1 (Atg6 in yeast), and Atg14.

In bulk autophagy in budding yeast, Atg17 is the main structural scaffold for the Atg1 complex. Atg17 is constitutively assembled with two small subunits, Atg29 and Atg31. The structure of the whole Atg17-Atg31-Atg29 complex was shown to be a 35-nm long S-shaped dimer, initially by crystallography and small angle x-ray scattering (SAXS) (10), and subsequently at $37-\AA$ A resolution by negative stain EM (11). The EM analysis corroborated the arrangement of subunits inferred from crystallography and SAXS. The S-shape is proposed to help scaffold high curvature vesicles for subsequent fusion into the phagophore, which is illustrated in Fig. 1. Another tethering complex, TRAPPIII, is also involved in the tethering of autophagic precursor vesicles. The 22- $\AA$ resolution EM structure of TRAPPIII (Fig. 2A) shows that it is rod-shaped and that the autophagy-specific subunit Trs 85 is located at one tip (12).

Subsequent EM analysis of the Atg17-31-29 complex established that the Atg13 subunit bound to the tips of the S-shape (13). The importance of this is that Atg13 is the bridge to the catalytic Atg1 subunit. Here, the EM analysis and other studies using crystallography (14) and SAXS (15) were mutually corroborative. These studies have shown key parts of the Atg1 complex are arranged in three-dimensions, but many question remain about the functions of the scaffolding elements and their interconnection with kinase regulation. It is widely assumed that the mammalian counterpart of Atg17, FIP200, will have a similar scaffolding function in the ULK1 complex. Sequence homology between FIP200 and Atg17 is so low, however, that it is not clear if the dimerization domain or Atg13 binding regions are conserved or not. The mammalian ULK1 complex is thus another important and as-yet untouched target for cryo-EM studies.

The three-dimensional structure of the human PI3KC3-C1 complex was first revealed at 28$\AA$ in a negative stain reconstruction (Fig. 2B) from our groups (16). EM revealed that the complex is conformationally variable (Fig. 2C). Nevertheless, it was possible to reconstruct the density for the predominant conformation. At this resolution, crystal structures cannot be reliably docked on the basis of the EM density alone. In addition to low resolution, limitations of negative stain reconstructions may include distortions (e.g. flattening) caused by the dehydration process. Moreover, the PI3KC3-C1 particles were preferentially oriented, such that the V-shape was presented face on in most views. In the worst cases, preferential orientation can result in the loss of projected views that are essential to accurately reconstruct the 3D object. This latter limitation can affect both negative stain and cryo-EM studies.

Tagging with maltose-binding protein (MBP) was used to locate the $\mathrm{N}$ - and $\mathrm{C}$-termini of the four subunits. Crystal structures were available for many of the domains of PI3KC3-C1, and homology models could be constructed for most of the others. This made it possible to place the crystallized domains within the density map for the full complex, despite the low resolution of the study. The accuracy of these placements was corroborated by a subsequent crystal structure of yeast PI3KC3-C2 determined at 4.4 A resolution (17). An analysis was also carried out for the human PI3KC3-C2 complex, in which the Atg14 subunit of complex 
I is replaced by UVRAG. PI3KC3-C2 functions in endosome maturation and in late stages of autophagy. The overall architecture of complexes I and II are nearly identical (16).

PI3KC3 has the shape of the letter "V", at least in its most populated conformation. The catalytic arm contains the Vps34 lipid kinase catalytic domain and the Vps15 kinase/ pseudokinase domain. These two domains are in contact with one another and form the outer tip of the "right-hand" arm. The "left-hand" arm consists of the central-coiled coil regions of BECN1 and Atg14 (or UVRAG in complex II), the WD40 domain of Vps15, and the BARA domain of BECN1. The junction of the $\mathrm{V}$ is formed by the HEAT repeats of Vps15, the $\mathrm{C} 2$ domain of Vps34, and the N-terminal regions of BECN1 and either Atg14 or UVRAG. Most of the known regulatory signals that impinge on $\mathrm{PI} 3 \mathrm{KC} 3-\mathrm{C} 1$ act on regions at the junction. This includes the recently characterized activating subunit NRBF2 (18). This suggests that there must be some mechanism for long-range communication between the base of $\mathrm{V}$ and the tip of the right-hand arm.

Single particle EM studies are challenged by conformational and compositional heterogeneity, but are also uniquely suited to detect and characterize such state variability, often at the heart of molecular function. During computational analysis, each image of the molecule (referred to as "particle") needs to be assigned angles (which define which view of the molecule the particle image corresponds to). If heterogeneity exists, the particle also needs to be assigned a certain state, out of the ensemble of existing states. The ability of single particle EM to carry out this "sorting" of states will ultimately dictate the accuracy and the resolution of the resulting structures. The success of these sorting procedures depends on the signal in the images, the nature of the heterogeneity, and the total amount of data. Some types of heterogeneity, such as the presence of intrinsically disordered regions (IDRs), are not amenable to subclassification because the conformation may differ in every particle. IDRs, which are common in autophagy protein in particular, will thus typically be invisible in 3D EM reconstructions. Both 2D and 3D sorting can be carried out. The EM analysis of the PI3KC3 complexes highlighted the internal flexibility of the complex. In addition to the V-shaped structure described above, at least two alternative conformations were visualized. In one, the two arms close up such that they are separated by about 45 degrees, as compared to about 90 degrees in the "classic" conformation (Fig. 2C). In the other, the Vps34 catalytic domain is dislodged from its position in the right arm and is only loosely tethered in the vicinity of the rest of the complex. EM allows us to directly visualize subpopulations of distinct conformations. Internally flexible complexes are sometimes considered "difficult" to analyze by EM, yet it the very ability of EM to visualize flexibility that makes it such an insightful tool.

The full power of high-resolution cryo-EM has not yet been realized for these two systems. Sample preparation for cryo-EM studies constitutes the major bottleneck for progress in structural characterization of many systems. Today's methodology for the preparation of a frozen-hydrated EM sample involves the thinning of the sample solution deposited on an EM grid to a layer that is just barely thicker than the size of the macromolecule under study. The rationale for this procedure has to do with the fact that cryo-EM images are limited by poor image contrast (low signal-to-noise ratio). This limitation itself stems from the need to minimize electron dose to reduce radiation damage, and the fact that atoms in biological 
systems (i.e. C, N, O) scatter similarly to the water background. Thus, reducing the amount of vitrified water around the molecule provides higher contrast due to lower noise. However, thinning of the solution creates air-water interfaces that are encountered by the molecules before the freezing process and have the potential to affect the integrity of the protein and even denature it. These limitations become more critical for less robust samples, and for macromolecules of smaller size. No magic, general solution yet exists for this problem, and optimization of the freezing procedures for the appropriate thickness of the ice and preservation of sample integrity is generally done one sample at a time. In some cases the use of surfactants, the mild crosslinking of the sample, or the use of more protein-friendly surfaces than the hydrophobic air-water interface (e.g. a thin, hydrophilic carbon film or a lipid monolayer) are some of the strategies so far used, sometimes in combination, to try to overcome the shortcomings of the present vitrification procedures. This is an area where clearly further research and ingenuity are needed. A general solution for cryo-EM sample preparation will be critical to make cryo-EM a more generally accessible technique to the biology community.

\section{mTORC1 and the upstream regulation of autophagy}

The inactivation of mTORC1 kinase activity in response to amino acid starvation is the main trigger for bulk autophagy. In experimental settings, autophagy is often induced by treatment with the mTORC1 inhibitor rapamycin. mTORC1 consists of the mTOR catalytic subunit and the regulatory subunits mLST8 and Raptor. The first cryo-EM structure of human mTORC1 was determined at $26 \AA$ resolution, and revealed a rhomboid dimer with a central cavity (19). The same structure was re-determined very recently at $5.9 \AA$ resolution (Fig. 2D) (20), highlighting the enormous technical advances made in cryo-EM in the past few years. A yeast Tor-Lst8 subcomplex structure was also recently reported at $6 \AA$ A resolution (21). Both of the new structures agree with respect to the nature of the mTOR dimer interface. The human mTORC1 shows that in the presence of rapamycin, a fourth protein, FKBP, is bound near the active site cleft of mTOR. Its presence is thought to sterically restrict the access of some substrate to the active site. This seems to explain how the drug rapamycin inhibits mTORC1. Yet it remains almost completely mysterious how, at the structural level, mTORC1 is regulated by physiological stimuli such as amino acids.

\section{Selective autophagy}

The "original" selective autophagy substrate is the precursor aminopeptidase I (prApe1) particle in budding yeast. The structure of this particle has been determined by negative stain EM at $19 \AA$ A resolution (Fig. 2E) (22). The x-ray structure of the mature particle, also a dodecamer, was determined at 2.5 and $2.8 \AA(22,23)$, and the negative stain EM structure at $24 \AA$ (23). The structures of the precursor and mature particle are sufficiently similar that the crystallographic dodecamer could be docked into the EM reconstruction of prApe1. The propeptides are involved in targeting prApe1 for transport to the vacuole. The structure shows that the geometry of propeptide presentation on the dodecamer surface is important for selective autophagic uptake (22). The cryo-EM structure of another autophagic cargo, $a-$ mannosidase I (Ams1) has been determined at $6.3 \AA$ A resolution (23). Ams1 is a tetramer and 
is considered a secondary cargo. Current thinking is that prApe1 dodecamers scaffold the Cvt vesicle, whilst other cargoes such as Ams1 may "come along for the ride" as passengers.

Selective autophagy in humans is directed by various autophagy adaptors that connect cargo, often ubiquitinated, to the autophagy machinery. These adaptors include p62, NBR1, optineurin, TAX1BP1, NDP52, TOLLIP, and RPN10. p62 is one of the best studied adaptors and consists of a PB1 domain, a KEAP1 interacting region (KIR), an LC3 interacting region (LIR), and a ubiquitin-binding UBA domain. The PB1 domain of p62 is responsible for oligomerization. In vitro, purified p62-PB1 forms helical filaments and tubes (24). Cryo-EM and helical reconstruction was used to determine the structures of two p62-PB1 assemblies at 10-11 ̊ resolution (Fig. 2F) (24). The crystal structure of a related PB1 domain was available and was used to interpret the reconstruction in molecular terms and identify residues involved in helical contacts. Surprisingly, the helical contacts seem to be mediated by electrostatic self-complementarity between two-fold related copies of the highly charged C-terminal extension of the PB1 domain. Mutation of these charged regions diminishes the extent of aggregates formed by overexpressed p62 in vivo (24). Other autophagy adaptors are not known to form helical assemblies at it seems unlikely that this is a general property of these adaptors. It seems more likely that the helical interface seen in the reconstruction is used physiologically to form smaller p62 oligomers that contribute to avidity.

\section{Structure of the autophagosome}

The biogenesis of the mammalian autophagosome was first examined in two influential EM tomographic studies $(25,26)$ of conventionally fixed NRK cells and mouse fibroblasts. In both studies, the nascent autophagosome (referred to as the phagophore/isolation membrane) is cradled almost entirely by a $\sim 500 \times 1000 \mathrm{~nm}$ cocoon of ER (Fig. 1). In mouse fibroblasts, a single connecting bridge was seen to link the phagophore and the ER, while multiple connections were seen in NRK cells. These interconnections appear to consist of clusters of high curvature tubular vesicles (27). Regardless of the number of the connections, a better understanding of the nature of the connections is critical to the mechanism of autophagosome biogenesis. Cryo x-ray tomography is one of the innovative approaches being taken, allowing imaging of whole cells (28). Better resolving the precise nature of the membrane connections is absolutely central to understanding how lipids are delivered to the growing autophagosome. This will require even more advanced applications of cellular cryoEM.

Cryo-electron tomography can potentially produce more detailed descriptions of the molecular nature of these connections, as it relies on the natural contrast of the protein components, rather than stain uptake within the fixed and resin-embedded sample. However, the difficulty in cryo-sectioning frozen hydrated cellular samples have precluded fast progress in the more general cases of cryo-electron tomography analysis (successes so far have been typically limited to cell edges where cryo-sectioning is not needed or for extracted organelles/cellular contents). A new approach that holds great promise to visualize intact regions inside cells is FIB milling, in which an ion beam is used to shed away cellular contents to generate a lamella thin enough for cryo-EM imaging and tomographic reconstruction. Imaging itself has improved with the use of energy filters and direct 
detectors, and most recently, the use of phase plate technology. Extraction of subtomogram features that can be classified and then averaged has started to produce structures of macromolecular assemblies in situ that approach $1 \mathrm{~nm}$ resolution in the most optimal cases. The limitation of the approach just described is that the region of the cell being characterize is typically only $\sim 200 \mathrm{~nm}$ thick. In the description of membrane trafficking events that involved features significantly larger, alternative EM approaches can provide very valuable complementary information that, although at lower resolution, cover very large cellular real state. Among them are block face-SEM and FIB SEM. Indeed, one study using block faceSEM has replicated the observation of multiple interconnections between the phagophore and the ER in NRK cells (29). The capability to produce a surface rendition of the sample by SEM, is coupled to a process in which epilayers of the sample are either removed by sectioning, of shedded using an ion beam. The former can be used for larger samples while the latter can chive higher resolution in the z-direction of smaller samples. $8 \mathrm{~nm}$ can already be achieved by FIB-SEM of whole cells, and better resolution could be in the horizon.

\section{Concluding remarks}

The recent technological breakthroughs in cryo-EM allowing both to produce near-atomic resolution structure of macromolecular assemblies, and to describe the cellular architecture with unprecedented detail, have only just begun to contribute to autophagy research but promise to have a high impact in our understanding of this essential cellular process. Single particle EM is uniquely suited to detect and characterize conformational complexity in macromolecular assemblies, a property bound to have a major role in the highly dynamic autophagic complexes. In the cellular context, where better resolving the nature of the autophagothitic membrane structures, such as the interconnections between the phagophore and the ER, is absolutely central to understanding how lipids are delivered to the growing autophagosome, cryo-tomography and FIB/SEM have the potential to shed unique light for our understanding of autophagy. Over the long term, advances in structural and cellular imaging by EM should eventually allow the docking of autophagic protein complexes into cellular cryo-EM tomograms of autophagosomes. This is already being achieved in studies of nuclear pores $(30,31)$, giving the autophagy imaging world a model to aspire to.

\section{Acknowledgments}

We thank Arthur Yeremenko and Lindsey Young for generating figures. The application of EM to autophagy in our labs is supported by grants P01 GM051487 (E.N. and J.H.H.) and R01 GM111730 (J.H.H.)

\section{References}

1. Wen X, Klionsky DJ. An overview of macroautophagy in yeast. J Mol Biol. 2016

2. Zaffagnini G, Martens S. Mechanisms of selective autophagy. J Mol Biol. 2016

3. Mancias JC, Kimmelman AC. Mechanisms of selective autophagy in normal physiology and cancer. J Mol Biol. 2016

4. Nixon RA. The role of autophagy in neurodegenerative disease. Nat Med. 2013; 19:983. [PubMed: 23921753]

5. Eskelinen EL, Reggiori F, Baba M, Kovacs AL, Seglen PO. Seeing is believing The impact of electron microscopy on autophagy research. Autophagy. 2011; 7:935. [PubMed: 21566462] 
6. Lamb CA, Yoshimori T, Tooze SA. The autophagosome: origins unknown, biogenesis complex. Nature Reviews Molecular Cell Biology. 2013; 14:759. [PubMed: 24201109]

7. Nogales E. The development of cryo-EM into a mainstream structural biology technique. Nat Methods. 2016; 13:24. [PubMed: 27110629]

8. Papinski D, Kraft C. Regulation of autophagy by signalling through the Atg1/ULK1 complex. J Mol Biol. 2016

9. Lin MG, Hurley JH. Structure and function of the ULK1 complex in autophagy. Curr Opin Cell Biol. 2016; 39:61. [PubMed: 26921696]

10. Ragusa MJ, Stanley RE, Hurley JH. Architecture of the Atg17 Complex as a Scaffold for Autophagosome Biogenesis. Cell. 2012; 151:1501. [PubMed: 23219485]

11. Chew LH, Setiaputra D, Klionsky DJ, Yip CK. Structural characterization of the Saccharomyces cerevisae autophagy regulatory complex Atg17-Atg31-Atg29. Autophagy. 2013; 9:1467. [PubMed: 23939028]

12. Tan D, et al. The EM structure of the TRAPPIII complex leads to the identification of a requirement for COPII vesicles on the macroautophagy pathway. Proc Natl Acad Sci U S A. 2013; 110:19432. [PubMed: 24218626]

13. Chew LH, et al. Molecular interactions of the Saccharomyces cerevisiae Atg1 complex provide insights into assembly and regulatory mechanisms. Autophagy. 2015; 11:891. [PubMed: 25998554]

14. Fujioka Y, et al. Structural basis of starvation-induced assembly of the autophagy initiation complex. Nat Struct Mol Biol. 2014; 21:513. [PubMed: 24793651]

15. Köfinger J, Ragusa MJ, Lee IH, Hummer G, Hurley JH. Solution structure of the Atg1 complex: Implications for the architecture of the phagophore assembly site. Structure. 2015; 23:809. [PubMed: 25817386]

16*. Baskaran S, et al. Architecture and Dynamics of the Autophagic Phosphatidylinostol 3-Kinase Complex. eLife. Dec 9.2014 3 Shows that the PI3KC3-C1 complex of autophagy is V-shaped, that the Vps15 and Vps34 kinase domains have extensive contact with one another, and that the complex has at least two modes of conformational variability.

17. Rostislavleva K, et al. Structure and flexibility of the endosomal Vps34 complex reveals the basis of its function on membranes. Science. 2015; 350

18. Young LN, Cho K, Lawrence R, Zoncu R, Hurley JH. Dynamics and architecture of the NRBF2containing phosphatidylinositol 3-kinase complex I of autophagy. Proc Natl Acad Sci U S A. 2016 in press.

19. Yip CK, Murata K, Walz T, Sabatini DM, Kang SA. Structure of the Human mTOR Complex I and Its Implications for Rapamycin Inhibition. Mol Cell. 2010; 38:768. [PubMed: 20542007]

20*. Aylett CHS, et al. STRUCTURAL BIOLOGY Architecture of human mTOR complex 1. Science. 2016; 351:48. Long-sought structure of mTORC1 shows details of assembly and dimerization, and offers insights into inhibition by rapamycin. [PubMed: 26678875]

21. Baretic D, Berndt A, Ohashi Y, Johnson CM, Williams RL. Tor forms a dimer through an Nterminal helical solenoid with a complex topology. Nature Communications. 2016; 7

22*. Su M-Y, et al. Structure of yeast Ape1 and its role in autophagic vesicle formation. Autophagy. $2015 ; 11: 1580$. This and ref. 23 show how the geometry of the major substrate of selective autophagy in yeast, prApe1, contributions to scaffolding its own uptake. [PubMed: 26208681]

23*. Bertipaglia C, et al. Higher-order assemblies of oligomeric cargo receptor complexes form the membrane scaffold of the Cvt vesicle. Embo Reports. 2016 See 22 above.

24. Ciuffa R, et al. The Selective Autophagy Receptor p62 Forms a Flexible Filamentous Helical Scaffold. Cell Reports. 2015; 11:748. [PubMed: 25921531]

25. Hayashi-Nishino M, et al. A subdomain of the endoplasmic reticulum forms a cradle for autophagosome formation. Nat Cell Biol. 2009; 11:1433. [PubMed: 19898463]

26. Yla-Anttila P, Vihinen H, Jokita E, Eskelinen EL. 3D tomography reveals connections between the phagophore and endoplasmic reticulum. Autophagy. 2009; 5:1180. [PubMed: 19855179]

27. Uemura T, et al. A Cluster of Thin Tubular Structures Mediates Transformation of the Endoplasmic Reticulum to Autophagic Isolation Membrane. Mol Cell Biol. 2014; 34:1695. [PubMed: 24591649] 
28. Duke EMH, et al. Imaging endosomes and autophagosomes in whole mammalian cells using correlative cryo-fluorescence and cryo-soft X-ray microscopy (cryo-CLXM). Ultramicroscopy. 2014; 143:77. [PubMed: 24238600]

29. Biazik J, Yla-Anttila P, Vihinen H, Jokitalo E, Eskelinen EL. Ultrastructural relationship of the phagophore with surrounding organelles. Autophagy. 2015; 11:439. [PubMed: 25714487]

$30 *$. Kosinski J, et al. Molecular architecture of the inner ring scaffold of the human nuclear pore complex. Science. 2016; 352:363. Along with ref. 31, this analysis of the nuclear pore complex shows what current technology is capable of and gives the structural autophagy field a model to aspire to. [PubMed: 27081072]

31*. Lin DH, et al. STRUCTURAL BIOLOGY Architecture of the symmetric core of the nuclear pore. Science. 2016; 352:308. See annotation to ref. 30.

32. Kim YG, et al. The architecture of the multisubunit TRAPP I complex suggests a model for vesicle tethering. Cell. 2006; 127:817. [PubMed: 17110339] 


\section{Highlights}

1. Different Electron microscopy modalities are needed for an understanding of autophagy

2. New mechanistic insights from single particle EM studies of the autophagy machinery

3. Prospects for resolving autophagic membrane structures by FIB-SEM and cryo-tomography 


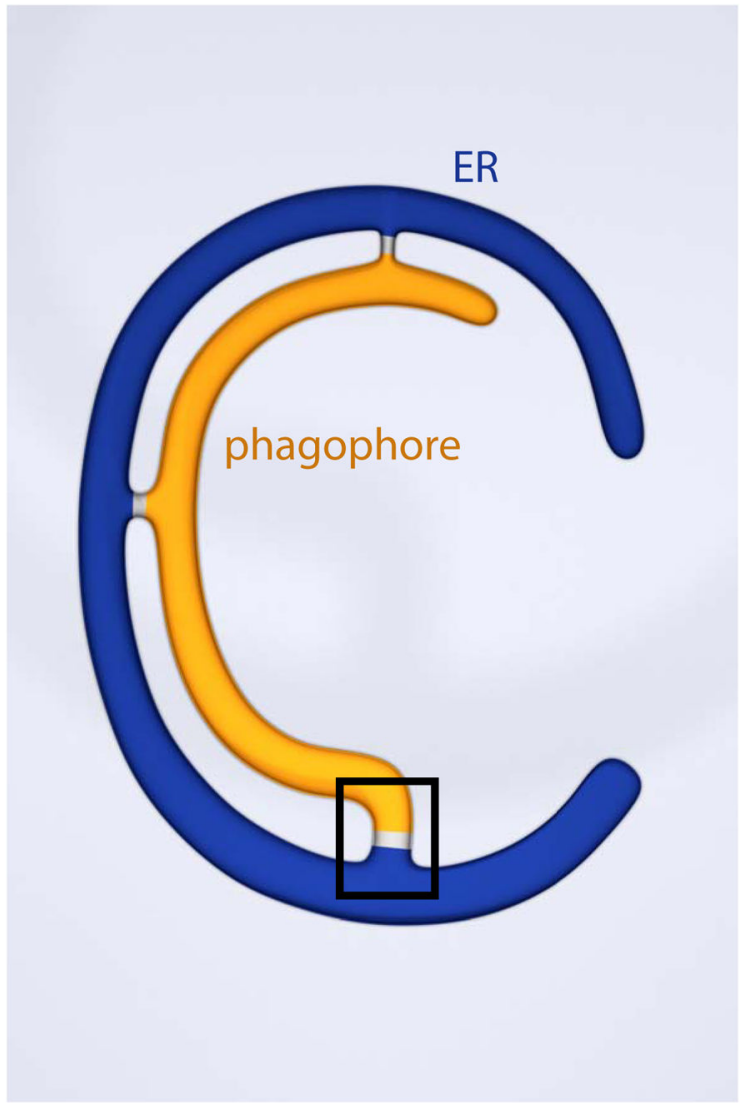

Figure 1. Autophagosome biogenesis

The mammalian autophagosome grows in a cradle of ER membrane known as the omegasome. This artist's rendering is inspired by the tomographic reconstructions of Hayashi-Nishino et al. (25). The black box indicates the region where higher resolution information is most critically needed. 
A

B
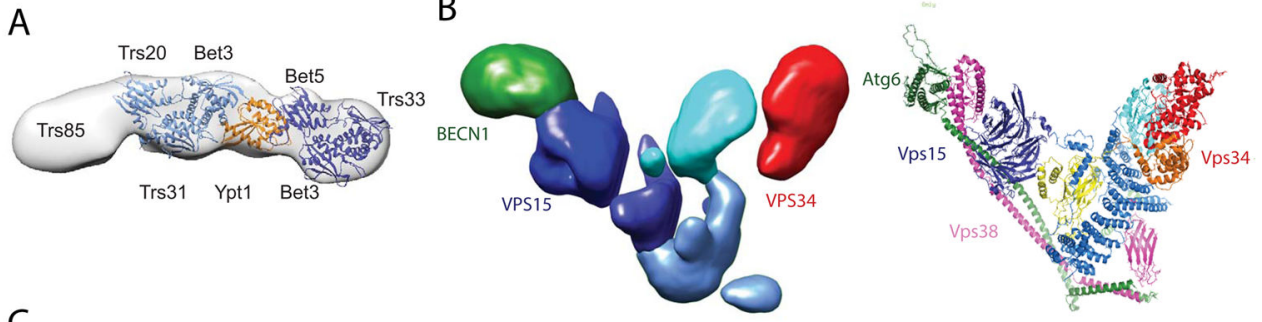

C
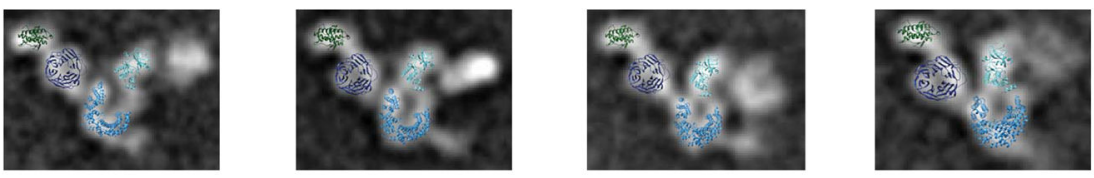

D

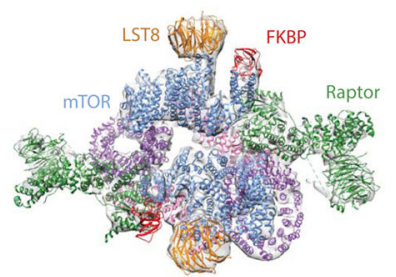

E

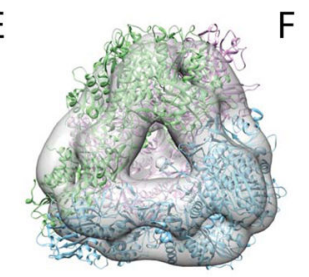

$\mathrm{F}$

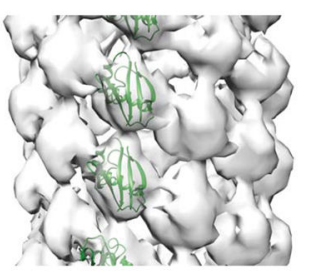

Figure 2. Electron microscopic reconstructions of assemblies involved in autophagy A. Structure of the TRAPIII complex (EMDB 5741) (12) docked with crystal structures of Bet3, Trs33, Bet5, Trs23 (from 2J3T) (32). B. Structure of the human PI3KC3-C1 complex (16) compared to the crystal structure of yeast PI3KC3-C2 (17). Left: BECN1 (green), VPS15 (blue), VPS34 (red), EMDB: 2846 Right: Atg6 (green), UVRag (pink), Vps15 (blue), Vps34 (C2 domain yellow, HELCAT orangered), PDB: 5DFZ. C. Conformational variability of PI3KC3-C1, reprinted from (16). D. Structure of mTORC1 (EMDB 3212, PDB 5FLC) (20), using crystal structure of CtRaptor (PDB 5EF5). Raptor (green), FKBP (red), mLST8 (orange), mTOR FAT/Kinase (blue), mTOR HEAT (purple). E. Structure of the mature Ape1 particle (EMDB 6265) (22) docked with three copies of the tetramer crystal structure (PDB 4R8F). F. Structure of the p62 PB1 domain filament (green, PDB: 2KKC) (EMDB 2936) (24). 